\title{
Covariant holography of a tachyonic accelerating universe
}

\author{
Alberto Rozas-Fernández ${ }^{1,2, a}$ \\ ${ }^{1}$ Instituto de Física Fundamental, Consejo Superior de Investigaciones Científicas, Serrano 121, 28006 Madrid, Spain \\ ${ }^{2}$ Institute of Cosmology and Gravitation, University of Portsmouth, Dennis Sciama Building, Portsmouth PO1 3FX, UK
}

Received: 15 May 2014 / Accepted: 31 July 2014 / Published online: 22 August 2014

(C) The Author(s) 2014. This article is published with open access at Springerlink.com

\begin{abstract}
We apply the holographic principle to a flat dark energy dominated Friedmann-Robertson-Walker spacetime filled with a tachyon scalar field with constant equation of state $w=p / \rho$, both for $w>-1$ and $w<-1$. By using a geometrical covariant procedure, which allows the construction of holographic hypersurfaces, we have obtained for each case the position of the preferred screen and have then compared these with those obtained by using the holographic dark energy model with the future event horizon as the infrared cutoff. In the phantom scenario, one of the two obtained holographic screens is placed on the big rip hypersurface, both for the covariant holographic formalism and the holographic phantom model. It is also analyzed whether the existence of these preferred screens allows a mathematically consistent formulation of fundamental theories based on the existence of an S-matrix at infinite distances.
\end{abstract}

\section{Introduction}

The holographic principle was put forward in 1993 [1,2] and asserts that all of the information contained in some region of space can be represented as a hologram, a theory located on the boundary of that region. This theory should contain at most one degree of freedom per Planck area. Since then, the holographic principle has been fruitfully developed, as in its most well-known implementation, the AdS/CFT correspondence [3], as well as in its connection with M-theory [4]. A cosmological version of the holographic principle was proposed in [5].

We shall study in this paper how the holographic principle applies to an accelerating universe filled with a tachyon scalar field with a constant equation of state $(\operatorname{EoS}) w=p / \rho$, for both the spacetimes of the region $-1<w<-1 / 3$ and the phantom domain $w<-1$. In order to do so, we shall make use of a covariant procedure [6] and the results will be confronted with those given by the holographic dark energy

\footnotetext{
a e-mail: a.rozas@iff.csic.es
}

model with the future event horizon as the infrared cutoff [7]. Our analysis is relevant in at least two aspects. On the one hand, dark energy should contain a large amount of the relevant degrees of freedom and hence, in order to constrain the EoS for dark energy, it is important to investigate whether such degrees of freedom are projected on the same boundary surfaces as those characterizing the remaining non-vacuum energy. On the other hand, a universe with constant EoS $w$ that accelerates indefinitely will exhibit a future event horizon [8] (see, however, [9]), presenting a challenge for string theories because it is not possible to construct a conventional S-matrix as the local observer inside his horizon is not able to isolate particles to be scattered. The emergence of an event horizon at the future, which would behave as a holographic screen, would aggravate this problem.

This paper can be outlined as follows. Section 2 contains the spacetime of a flat Friedmann-Robertson-Walker (FRW) universe filled with a tachyon scalar field in the region $w>$ -1 . In Sect. 3 a covariant formalism is used to derive the holographic preferred screens that correspond to the spacetime presented in Sect. 2. In Sect. 4 we discuss the covariant holography of a flat tachyonic phantom energy scenario. In Sect. 5 , the dark and phantom holographic dark energy models are constructed for a flat geometry in order to insert holographic screens in terms of the future event horizon [7] or the horizon at the big rip [10]. The conclusions are drawn in Sect. 6.

\section{The spacetime of a universe filled with tachyonic dark energy}

The explanation of dark energy is a central preoccupation of present-day cosmology. In the $\Lambda \mathrm{CDM}$ paradigm, in which the cosmological constant accounts for the acceleration of the universe, the universe would asymptotically tend to the de Sitter spacetime whose holographic properties have already been studied in some depth $[11,12]$. However, the dark energy could perfectly be dynamical in nature, even favoured over the cosmological constant [13]. 
If we consider the tachyon as a dark energy candidate, the spacetime structure that results presents some holographic properties that have not been considered yet and that deserve our attention.

The fact that the tachyon can act as a source of dark energy with different potential forms have been widely discussed in the literature [14-20]. The tachyon can be described by an effective field theory corresponding to a tachyon condensate in a certain class of string theories with the following effective action [21-23]:

$S=\int d^{4} x \sqrt{-g}\left[\frac{R}{16 \pi G}-V(\phi) \sqrt{1+g^{\mu \nu} \partial_{\mu} \phi \partial_{\nu} \phi}\right]$,

where $V(\phi)$ is the tachyon potential and $R$ the Ricci scalar. The physics of tachyon condensation is described by the above action for all values of $\phi$ provided the string coupling and the second derivative of $\phi$ are small.

The corresponding energy-momentum tensor of the tachyon field has the form

$T_{\mu \nu}=\frac{V(\phi) \partial_{\mu} \phi \partial_{\nu} \phi}{\sqrt{1+g^{\alpha \beta} \partial_{\alpha} \phi \partial_{\beta} \phi}}-g_{\mu \nu} V(\phi) \sqrt{1+g^{\alpha \beta} \partial_{\alpha} \phi \partial_{\beta} \phi}$.

Let us now consider a spatially flat FRW spacetime with line element

$d s^{2}=-d t^{2}+a(t)^{2} d \mathbf{x}^{2}$

in which $a(t)$ is the scale factor. The Friedmann equations then read

$$
\begin{aligned}
& H^{2} \equiv\left(\frac{\dot{a}}{a}\right)^{2}=\frac{8 \pi G \rho_{t}}{3} \\
& \frac{\ddot{a}}{a}=-\frac{4 \pi G\left(\rho_{t}+3 p_{t}\right)}{3}
\end{aligned}
$$

where the energy density $\rho_{t}$ and the pressure $p_{t}$ are given by

$$
\begin{aligned}
& \rho_{t}=-T_{0}^{0}=\frac{V(\phi)}{\sqrt{1-\dot{\phi}^{2}}}, \\
& p_{t}=T_{i}^{i}=-V(\phi) \sqrt{1-\dot{\phi}^{2}},
\end{aligned}
$$

and the dot stands for the derivative with respect to cosmic time.

From Eqs. (6) and (7) we obtain the tachyon EoS parameter

$w=\frac{p_{t}}{\rho_{t}}=\dot{\phi}^{2}-1$

and we shall consider $w$ in what follows to obey $w>-1$. We shall also restrict ourselves to consider a description of the current cosmic situation where it is assumed that the tachyon component largely dominates and therefore we shall disregard the non-relativistic and relativistic components of the matter density and pressure.

If we assume a linear time-dependence of the tachyon field $\phi$ and hence constancy of the parameter $w$, then the general expression for $a(t)$ can be written as

$a(t)=\left[a_{0}^{3(1+w) / 2}+\frac{3}{2}(1+w)\left(t-t_{0}\right)\right]^{2 /[3(1+w)]}$

where $a_{0}$ is the initial value of the scale factor at the initial time $t_{0}>0$. This solution describes an accelerating universe in the interval $-1<w<-1 / 3$. In order to facilitate the study of the holographic properties of the tachyonic spacetime, it is best to express solution (9) in terms of the conformal time

$\eta=\int \frac{\mathrm{d} t}{a(t)}=\frac{2 a^{(1+3 w) / 2}}{1+3 w}$

Note that $-\infty<\eta<0$ for $w<-1 / 3$. Therefore, the scale factor in terms of the conformal time now reads

$a(\eta)=\left[\frac{(1+3 w) \eta}{2}\right]^{2 /(1+3 w)}$.

\section{Covariant holography in a tachyonic accelerating universe}

In this section we shall carry out the study of the holographic properties of the spacetime presented in Sect. 2, following the covariant formalism developed in [6] for general spacetimes. We shall start first by drawing the Penrose diagram for our tachyonic asymptotic spacetime and then we shall construct the embedded holographic hypersurfaces (screens), which are surfaces on which the information in the spacetime bulk can be encoded at less than one bit per Planck area [1,2]. In order to construct screens, we must slice the spacetime into a family of light cones centered at $r=0$ that can be parameterised by time and then identify in which direction to project among the two inequivalent null projections, which go along past or future-directed light cones.

The Penrose diagram is constructed by mapping our FRW spacetime on a part of the Einstein static universe [24], whose causal structure is that of an infinite cylinder $R \times S^{3}$, and determining the regions of it that are conformal to our FRW spacetime. In the resulting Penrose diagram, every point represents a $S^{2}$ sphere and each diagonal line represents a light cone. The two inequivalent null slices can be represented by the ascending and descending families of diagonal lines. We then proceed to identify the apparent horizons, which are defined geometrically as the spheres (hypersurfaces) at which at least one pair (past or future) of orthogonal null congruences has zero expansion. These horizons will divide 
the spacetime into normal, trapped and anti-trapped regions $[6,24]$.

We shall finally determine the preferred and optimal (if any) screen hypersurfaces which are going to encode all the information in the universe. A preferred screen is a surface in which the expansion of all projected null hypersurfaces becomes zero at every point [6]. If the expansions of both independent pairs of orthogonal families of light-rays vanish on one of the preferred screens, it becomes an optimal screen [6].

A flat FRW spacetime is described, in terms of the conformal time $\eta$, by a metric of the form

$d s^{2}=a(\eta)^{2}\left(-d \eta^{2}+d r^{2}+r^{2} d \Omega_{2}^{2}\right)$,

where $0<r<\infty$, and $d \Omega_{2}^{2}=d \theta^{2}+\sin ^{2} \theta d \phi^{2}$ is the metric on the unit $S^{2}$ sphere, with $0<\theta<\pi$ and $0<\phi<2 \pi$. This metric can be reduced to a more convenient form [24] by defining some new coordinates, $p$ and $q$, such that $t^{\prime}=p+q$ and $r^{\prime}=p-q$. This allows the metric (12) to be expressed in a form which is conformal to that of Minkowski space in spherical coordinates, and hence locally identical to that of the Einstein static universe

$$
\begin{aligned}
d s^{2}= & \frac{1}{4} a^{2} \sec ^{2}\left[\frac{1}{2}\left(t^{\prime}+r^{\prime}\right)\right] \sec ^{2}\left[\frac{1}{2}\left(t^{\prime}-r^{\prime}\right)\right] \\
& \times\left[-\left(d t^{\prime}\right)^{2}+\left(d r^{\prime}\right)^{2}+\sin ^{2} r^{\prime} d \Omega_{2}^{2}\right],
\end{aligned}
$$

where $-\pi<t^{\prime}+r^{\prime}<\pi,-\pi<t^{\prime}-r^{\prime}<\pi, r^{\prime} \geq 0$. The new coordinates $r^{\prime}$ and $t^{\prime}$ are related to the original coordinates $\eta$ and $r$ by

$\eta=\frac{1}{2} \tan \left[\frac{1}{2}\left(t^{\prime}+r^{\prime}\right)\right]+\frac{1}{2} \tan \left[\frac{1}{2}\left(t^{\prime}-r^{\prime}\right)\right]$

$r=\frac{1}{2} \tan \left[\frac{1}{2}\left(t^{\prime}+r^{\prime}\right)\right]-\frac{1}{2} \tan \left[\frac{1}{2}\left(t^{\prime}-r^{\prime}\right)\right]$.

Obviously, our flat FRW spacetime filled with a tachyon scalar field and whose EoS lies in the range $-1<w<-1 / 3$ can be mapped into the part of the Einstein static universe determined by the values taken by $\eta$ in the interval $-\infty<\eta<0$, which corresponds to the ranges $-\pi<t^{\prime}<0$ and $0<r^{\prime}<\pi$. From these, the resulting Penrose diagram follows after determining the region of the Einstein static space which is conformal to our tachyonic flat spacetime.

The parts of the Einstein static cylinder which are conformal to the tachyonic flat FRW spacetime for $-1<w<$ $-1 / 3$ are shown in Fig. 1. The conformal region runs from $t^{\prime}=0$ to an extreme $t^{\prime}<0$. The corresponding Penrose diagram is plotted in Fig. 2.

Now, following the prescription given in [6], we can construct the holographic screens in our tachyonic flat FRW universe. The apparent horizon is given by $\eta=2 r /(1+3 w)$. The interior of the apparent horizon, $\eta \geq 2 r /(1+3 w)$, can be projected along future light cones centered at $r=0$, or by

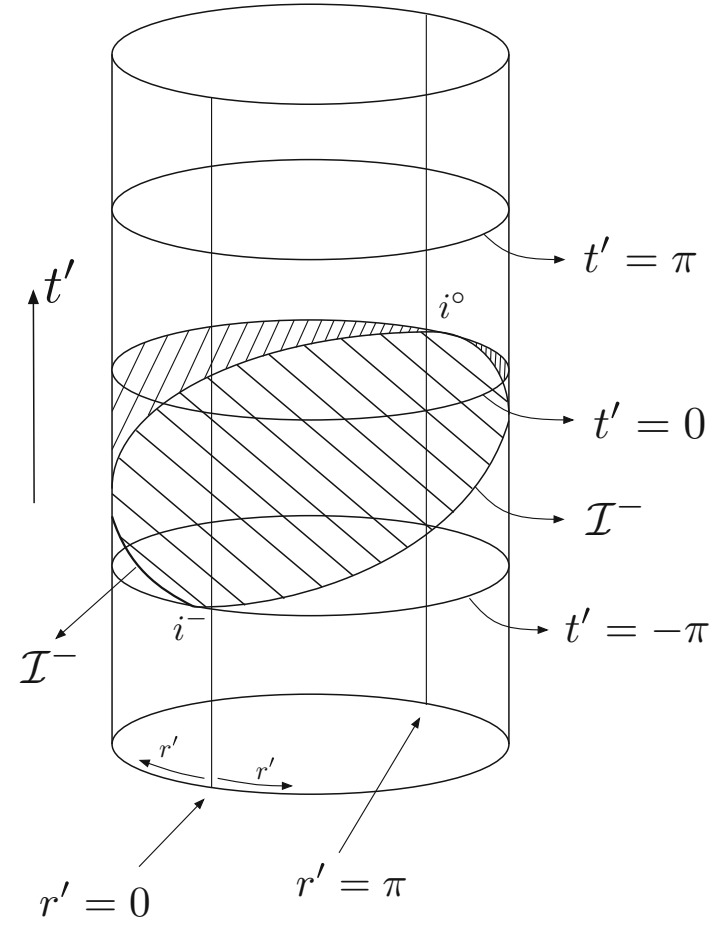

Fig. 1 The flat FRW spacetime filled with a tachyonic scalar field is conformal to the Einstein static universe for the EoS range $-1<w<$ $-1 / 3$. This representation looks similar to that of the de Sitter space, although it covers a larger $t^{\prime}$-interval

means of a space-like projection, onto the apparent horizon. The exterior, $\eta \leq 2 r /(1+3 w)$, can also be projected by future light cones, but in the opposite direction, onto the apparent horizon. Alternatively, the entire flat tachyonic universe can be projected along past light cones onto the past null infinity. The two holographic preferred screens that encode the entire spacetime, given by the apparent horizon $\eta=2 r /(1+3 w)$ and the past null infinity $\mathcal{I}^{-}$, are plotted in Fig. 2.

\section{Covariant holography in a tachyonic phantom universe}

Phantom dark energy $[25,26]$ has already confirmed its validity as a dark energy candidate [27-30]. Moreover, Planck's latest results [31] plus WMAP low- $l$ polarisation (WP), when combined with Supernova Legacy Survey (SNLS) data, favor the phantom domain $(w<-1)$ at $2 \sigma$ level for a constant $w$

$w=-1.13_{-0.14}^{+0.13}(95 \% ;$ Planck $+W P+S N L S)$,

while the Union2.1 compilation of Type Ia supernovae ( $\mathrm{SNe}$ Ia) is more consistent with a cosmological constant $(w=$ -1 ). If we combine Planck+WP with measurements of $H_{0}$ [32], we get for a constant $w$ 


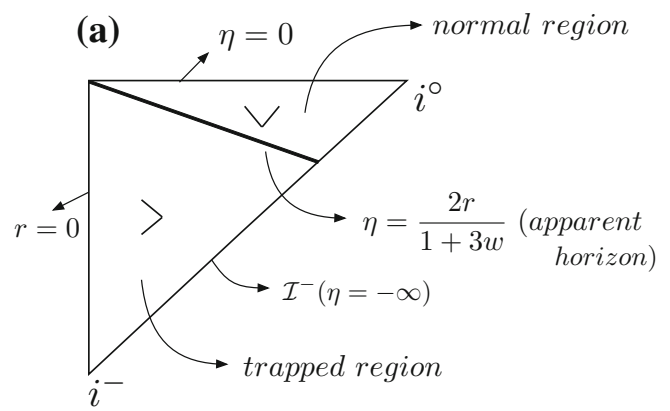

(b)

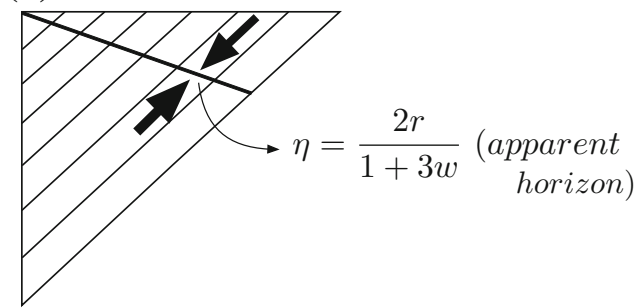

(c)

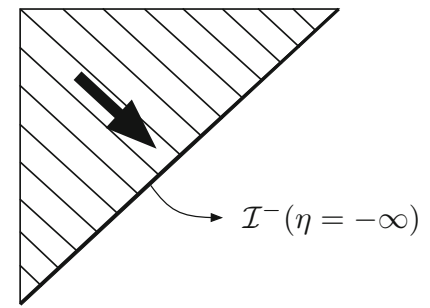

Fig. 2 Penrose diagram of a flat FRW universe filled with a tachyonic scalar field for the range $-1<w<-1 / 3$. The apparent horizon, $\eta=2 r /(1+3 w)$, divides the spacetime into a normal and a trapped region (a). The information contained in the universe can be projected along future light cones onto the apparent horizon (b), or along past light cones onto past null infinity $\mathcal{I}^{-}(\mathbf{c})$. Both are preferred screenhypersurfaces

$w=-1.24_{-0.19}^{+0.18}$

which is in tension with $w=-1$ at more than the $2 \sigma$ level. Also, for the SNLS3 and the Pan-STARRS1 survey (PS1 SN) data sets, the combined SNe Ia + Baryon acoustic oscillations $(\mathrm{BAO})+$ Planck data yield a phantom equation of state at $\sim 1.9 \sigma$ confidence [33]. The above observational results, in addition to theoretical motivations, are compelling enough to justify the study of the phantom sector in more depth.

The phantom regime, which implies a violation of the dominant energy condition

$p_{t}+\rho_{t}=\frac{V(\phi) \dot{\phi}^{2}}{\sqrt{1-\dot{\phi}^{2}}}<0$,

can be obtained by Wick rotating the tachyon field so that $\phi \rightarrow i \Phi$, where the field $\Phi$ can be viewed as an axion tachyon field [34], as the scale factor $a(t)$ and the field potential $V(\Phi)$ keep being positive. In this phantom case, the solution of Eqs. (4) and (5) for the scale factor yields [35].

$$
a(t)=\left[a_{0}^{3(1-|w|) / 2}+\frac{3}{2}(1-|w|)\left(t-t_{0}\right)\right]^{2 /[3(1-|w|)]},
$$

which in terms of the conformal time

$$
\eta=\int \frac{\mathrm{d} t}{a(t)}=\frac{2}{(1-3|w|) a^{(1-3|w|) / 2}}
$$

becomes

$a(\eta)=\left[\frac{(1-3|w|) \eta}{2}\right]^{2 /(1-3|w|)}$.

It is worth noting that in this phantom case $\eta$ runs from $\eta=-2 /(3|w|-1) a_{0}^{(3|w|-1) / 2} \equiv \eta_{0}<0$ at $t=t_{0}$, to $\eta=0$ at the big rip when

$t \equiv t_{b r}=t_{0}+\frac{2}{3(|w|-1) a_{0}^{3(|w|-1) / 2}}=t_{0}-\eta_{0}$,

to finally reach positive infinity as $t \rightarrow \infty$, therefore the interval is $\eta_{0}<\eta<+\infty$.

The field potential is given by [35]

$$
V(\Phi)=\frac{3 \sqrt{|w|}}{8 \pi G\left[a_{0}^{-3(|w|-1) / 2}-\frac{3}{2} \sqrt{|w|-1}\left(\Phi-\Phi_{0}\right)\right]^{2}},
$$

with $\Phi_{0} \rightarrow-i \phi_{0}$. We note that both this potential and the phantom tachyon energy density,

$$
\rho_{t}=\frac{3}{8 \pi G\left[a_{0}^{-3(|w|-1) / 2}-\frac{3}{2}(|w|-1) t\right]^{2}},
$$

increase with time up to blowing up at $t=t_{b r}$, to steadily decrease toward zero thereafter.

However, in order for this description to be applicable also after the big rip barrier at $t=t_{b r}$, such that the scale factor remains real and positive in that region, not all values of $w$ are allowed but only those that satisfy the discretization condition [10]

$w=-\frac{1}{3}\left(1+\frac{2 n+3}{n+1}\right), \quad n=0,1,2, \ldots$

Similarly to what we did in Sect. 2, we locally obtain the metric (12) for the Einstein static universe, where $\eta$ and $r$ are given by Eqs. (14) and (15).

Hence, the flat spacetime filled with a phantom tachyonic field we have just considered, which has an $\operatorname{EoS} p=w \rho$ with $-\infty<w<-1$, can also be mapped into those parts of the cylindric Einstein static universe which are determined by the values of conformal time $\eta$ we have discussed above. We 


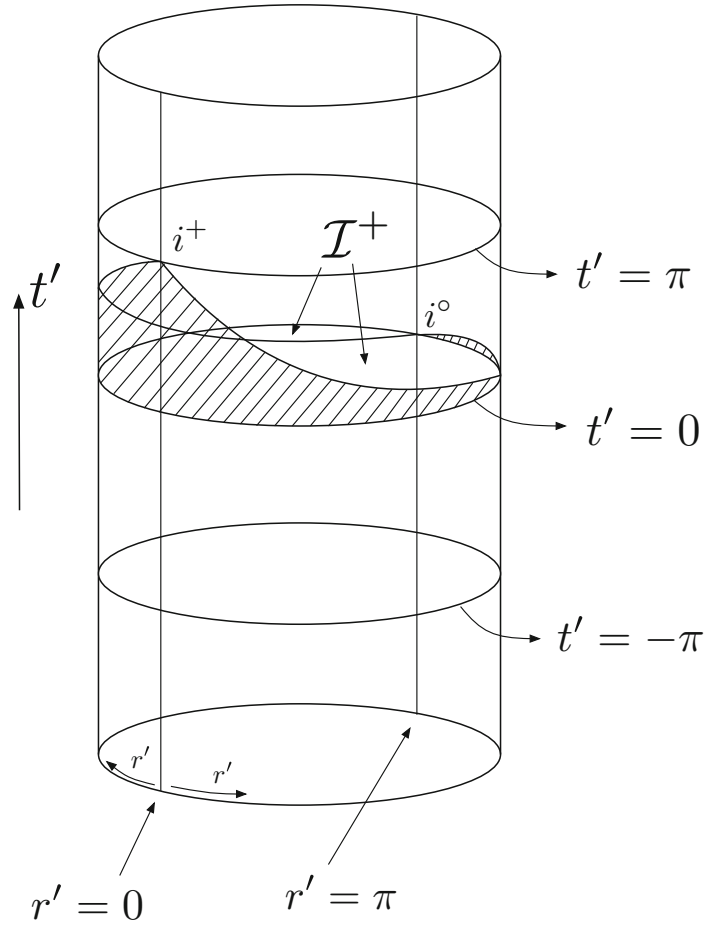

Fig. 3 The flat FRW spacetime filled with a tachyonic phantom energy scalar field is conformal to the Einstein static universe for the range $-\infty<w<-1$. This flat space looks similar to that of the de Sitter space $(p=-\rho)$, although it covers a larger $t^{\prime}$-interval

can see that the part $\eta_{0}<\eta<0$ of the whole interval $\eta_{0}<$ $\eta<+\infty$ will correspond to a subinterval, which depends on $w$, of the range $-\pi<t^{\prime}<0$ and $0<r^{\prime}<\pi$, and the part $0<\eta<+\infty$ will correspond to the range $0<t^{\prime}<\pi$ and $0<r^{\prime}<\pi$. This mapping is depicted in Fig. 3 and its resulting Penrose diagram is shown in Fig. 4.

\section{Holographic dark energy models}

Based on the holographic bound on the entropy $[1,2,36]$ and on the validity of effective local quantum field theory in a box of size L, Cohen et al. [37] suggested a relationship between the ultraviolet and the infrared cutoffs due to the limit set by the formation of a black hole. This led Li [7] to propose a most popular model of holographic dark energy (HDE) that can explain the accelerated expansion of the universe and in which the infrared cutoff is taken to be the observerdependent future event horizon which makes the holographic screen. This model is in good agreement with observational data [38-44] but has attracted some criticisms, known as the causality and circular logic problems [45]. Nevertheless, Li [46] has recently proposed a new HDE model with action principle in which these problems appear to be no longer present and the evolution of universe only depends on the present state of universe and the future event horizon cutoff automatically follows from the equations of motion. This new (a)
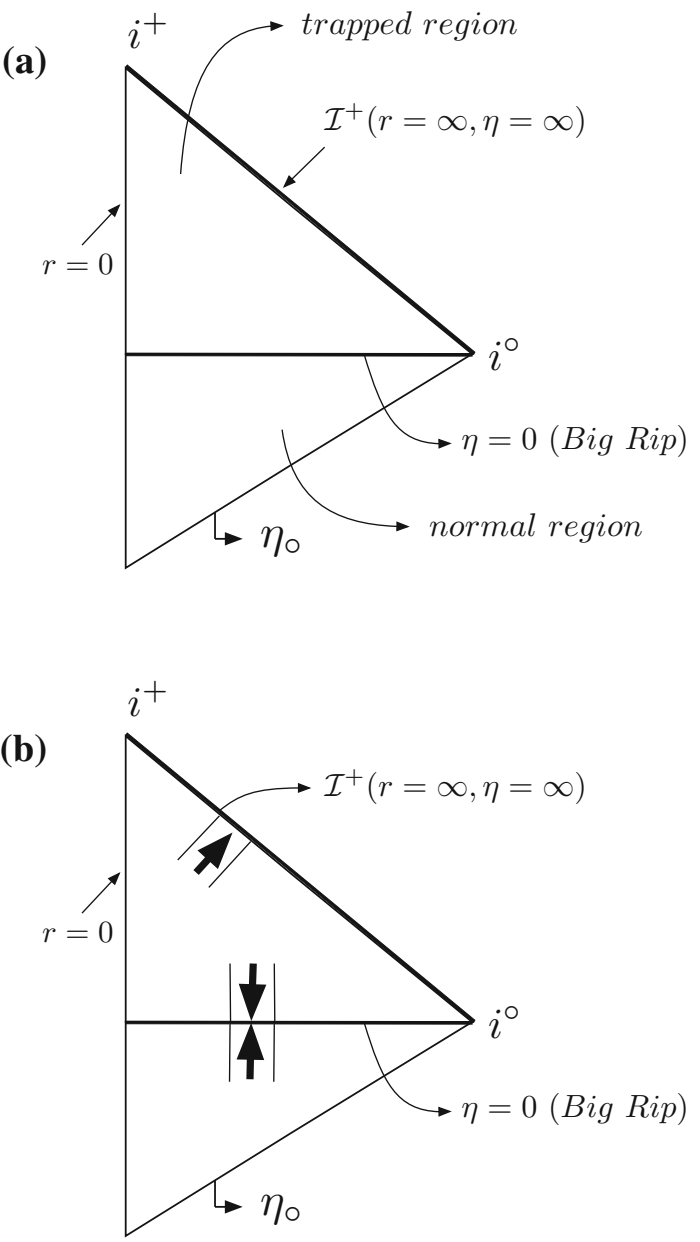

Fig. 4 Penrose diagram of a flat FRW universe filled with a tachyon phantom scalar field for $-\infty<w<-1$. The apparent horizon, also located at $\eta=2 r /(1+3 w)$, divides the spacetime into a normal and a trapped region (a). The information contained in the universe can be projected along future light cones from the normal region or along past light cones from the trapped region, both onto the apparent big rip horizon. It can also be projected along future light cones onto the future null infinity $\mathcal{I}^{+}(\mathbf{b})$. Both the big rip and $\mathcal{I}^{+}$are preferred screenhypersurfaces

HDE also complies well with the most recent observational constraints [47].

In a flat dark energy dominated FRW universe, Li's model [7] is based on the following relation between the Hubble parameter $H=\dot{a} / a$ and the size of the future event horizon $R_{h}$

$H^{2}=\frac{\dot{a}^{2}}{a^{2}}=\frac{8 \pi G \rho_{t}}{3}=\frac{c^{2}}{R_{h}^{2}}$,

where $R_{h}=a(t) \int_{t}^{\infty} \mathrm{d} t^{\prime} / a\left(t^{\prime}\right)$ is the proper size of the future event horizon which plays the role of the holographic screen and $c$ is a numerical parameter of order unity which is related to $w$ by $w=-(1+2 / c) / 3$. If we now express the scale factor given by Eq. (9) as a function of $c$ 
$a=\left[a_{0}^{(c-1) / c}+\frac{c-1}{c}\left(t-t_{0}\right)\right]^{c /(c-1)}=T(t)^{c /(c-1)}$,

the proper size of the future event horizon is then given by

$R_{h}=-c T(t)^{c /(c-1)}\left(\left.T\left(t^{\prime}\right)^{-1 /(c-1)]}\right|_{t} ^{\infty}\right)$.

Obviously, if $w>-1$ (i.e. $c>1$ ) then $R_{h}=c T(t)$, which is finite for finite $t$. Therefore, Li's model is only well defined when $w>-1$.

In the phantom case of Sect. $4, w<-1$ (i.e. $c<1$ ), and the proper size of the future event horizon inexorably becomes infinity, so we may say that it will vanish for phantom energy.

Since Eq. (26) is not well defined for $c<1$ as it leads to $H=0, \mathrm{Li}$ [7] argued that holographic phantom models were not viable. However, in the phantom scenario we should use instead of Eq. (26) the following [10]

$H_{\mathrm{ph}}^{2}=\frac{\dot{a}^{2}}{a^{2}}=\frac{8 \pi G \rho}{3}=\frac{c^{2}}{R_{b r}^{2}}$

where

$R_{b r}=a(t) \int_{t}^{t_{b r}} \frac{\mathrm{d} t^{\prime}}{a\left(t^{\prime}\right)}$

is the proper size of the future event horizon for the holographic phantom model, being $t_{b r}$ the time at which the big rip takes place.

For the tachyon phantom model Eq. (30) yields

$R_{b r}=c\left[a_{0}^{3(1-|w|) / 2}+\frac{3}{2}(1-|w|)\left(t-t_{0}\right)\right]^{2 /[3(1-|w|)]}$.

We have then seen that Eq. (26) is no longer valid for a covariant holographic description of an accelerating universe and that the appropriate holographic screens for the covariant specification are the one at the big rip hypersurface and the one at the future null infinity $\mathcal{I}^{+}$(see Sect. 4).

\section{Conclusions}

We have considered the holography of a flat FRW dark energy dominated universe in which the cause of its accelerated expansion is due to the presence of tachyon scalar field with constant EoS $w$. In order to do so, we have applied a covariant formalism [6] and then have compared the results with those obtained by the HDE model with the future event horizon as the infrared cutoff $[7,46]$.

The more general covariant formalism gives rise to two different holographic preferred screens. In the dark energy case $(w>-1)$ these are located at the apparent horizon $\eta=$ $2 r /(1+3 w)$ and at the past null infinity $\mathcal{I}^{-}$. On the other hand, in the phantom energy scenario $(w<-1)$ one is also located at the apparent horizon, which is the big rip hypersurface in this case, and the other at the future null infinity $\mathcal{I}^{+}$. When we establish the comparison of these results with the ones obtained by using the HDE model $[7,46]$, whose holographic screen is positioned at the future event horizon, we see that the former allow the definition of fundamental theories based on the existence of a S-matrix at infinite distances, at least when one approaches $\mathcal{I}^{-}$or $\mathcal{I}^{+}$.

There is in addition an apparent contradiction between the implications from the covariant treatment of phantom holography and the fact that phantom energy is characterized by a negative temperature [48]. We may be led to think that if the preferred holographic screens for phantom energy are located at the big rip and the future null infinity, then the entropy that should be associated with that phantom fluid would be negative definite, implying a definite positive temperature. However, this would be mistaken because the entropy involved in this case is the one defined by the surface area of the future preferred holographic screen, given in this case by Eq. (31).

The relevant entropy would actually coincide with the entropy of entanglement [49] and would be given by

$S_{E n t}=\left.\alpha R_{b r}^{2}\right|_{t>t_{b r}}$

where $\alpha$ is a constant of order unity. In order to calculate the entropy of entanglement we have used the equivalence between the regions before and after the big rip hypersurface. In this case, we have integrated out the region before that surface. This entanglement entropy is definite positive and increases with time, leading again to the conclusion that the temperature of a phantom fluid is definite negative.

Acknowledgments The author is grateful to Andrea Maselli for help with the plots. This work was supported by the 'Fundación Ramón Areces' and Ministerio de Economía y Competitividad (Spain) through project number FIS2012-38816.

Open Access This article is distributed under the terms of the Creative Commons Attribution License which permits any use, distribution, and reproduction in any medium, provided the original author(s) and the source are credited.

Funded by $\mathrm{SCOAP}^{3}$ / License Version CC BY 4.0.

\section{References}

1. Gerard 't Hooft, in Salamfest (1993), p. 284

2. L. Susskind, J. Math. Phys. 36, 6377 (1995). doi:10.1063/1.531249

3. J.M. Maldacena, Adv. Theor. Math. Phys. 2, 231 (1998)

4. P. Horava, Phys. Rev. D59, 046004 (1999). doi:10.1103/PhysRevD. 59.046004

5. W. Fischler, L. Susskind (1998). arXiv:hep-th/9806039

6. R. Bousso, Rev. Mod. Phys. 74, 825 (2002). doi:10.1103/ RevModPhys.74.825

7. M. Li, Phys. Lett. B603, 1 (2004). doi:10.1016/j.physletb.2004.10. 014 
8. X.-G. He (2001). arXiv:astro-ph/0105005

9. P.F. Gonzalez-Diaz, Phys. Lett. B522, 211 (2001). doi:10.1016/ S0370-2693(01)01305-3

10. P.F. Gonzalez-Diaz, Grav. Cosmol. 12, 29 (2006)

11. A. Karch, JHEP 0307, 050 (2003)

12. M. Alishahiha, A. Karch, E. Silverstein, JHEP 0506, 028 (2005). doi:10.1088/1126-6708/2005/06/028

13. L. Samushia, B.A. Reid, M. White, W.J. Percival, A.J. Cuesta et al., Mon. Not. Roy. Astron. Soc. 429, 1514 (2013)

14. J. Bagla, H.K. Jassal, T. Padmanabhan, Phys. Rev. D67, 063504 (2003). doi:10.1103/PhysRevD.67.063504

15. T. Padmanabhan, Phys. Rev. D66, 021301 (2002). doi:10.1103/ PhysRevD.66.021301

16. G.W. Gibbons, Phys. Lett. B537, 1 (2002). doi:10.1016/ S0370-2693(02)01881-6

17. A.V. Frolov, L. Kofman, A.A. Starobinsky, Phys. Lett. B545, 8 (2002). doi:10.1016/S0370-2693(02)02582-0

18. Y. Shao, Y.X. Gui, W. Wang, Mod. Phys. Lett. A22, 1175 (2007). doi:10.1142/S0217732307021809

19. G. Calcagni, A.R. Liddle, Phys. Rev. D74, 043528 (2006). doi:10. 1103/PhysRevD.74.043528

20. E.J. Copeland, M.R. Garousi, M. Sami, S. Tsujikawa, Phys. Rev. D71, 043003 (2005). doi:10.1103/PhysRevD.71.043003

21. E. Bergshoeff, M. de Roo, T. de Wit, E. Eyras, S. Panda, JHEP 0005, 009 (2000)

22. A. Sen, J. Math. Phys. 42, 2844 (2001). doi:10.1063/1.1377037

23. A. Sen, Int. J. Mod. Phys. A20, 5513 (2005). doi:10.1142/ S0217751X0502519X

24. S. W. Hawking, G.F.R. Ellis, The Large Scale Structure of SpaceTime (Cambridge University Press, Cambridge 1973)

25. R. Caldwell, Phys. Lett. B545, 23 (2002). doi:10.1016/ S0370-2693(02)02589-3

26. A.A. Starobinsky, Grav. Cosmol. 6, 157 (2000)

27. S.M. Carroll, M. Hoffman, M. Trodden, Phys. Rev. D68, 023509 (2003). doi:10.1103/PhysRevD.68.023509

28. P. Singh, M. Sami, N. Dadhich, Phys. Rev. D68, 023522 (2003). doi:10.1103/PhysRevD.68.023522

29. J.M. Cline, S. Jeon, G.D. Moore, Phys. Rev. D70, 043543 (2004). doi:10.1103/PhysRevD.70.043543

30. M. Sami, A. Toporensky, Mod. Phys. Lett. A19, 1509 (2004). doi:10.1142/S0217732304013921
31. P.A.R. Ade, et al., A\&A (2014). doi:10.1051/0004-6361/ 201321591

32. A.G. Riess, L. Macri, S. Casertano, H. Lampeitl, H.C. Ferguson et al., Astrophys. J. 730, 119 (2011). doi:10.1088/0004-637X/732/2/ 129, doi:10.1088/0004-637X/730/2/119

33. D.L. Shafer, D. Huterer, Phys. Rev. D89, 063510 (2014). doi:10. 1103/PhysRevD.89.063510

34. P.F. Gonzalez-Diaz, Phys. Rev. D69, 063522 (2004). doi:10.1103/ PhysRevD.69.063522

35. P.F. Gonzalez-Diaz, TSPU Vestnik 44N7, 36 (2004)

36. P. Gonzalez-Diaz, Phys. Rev. D27, 3042 (1983). doi:10.1103/ PhysRevD.27.3042

37. A.G. Cohen, D.B. Kaplan, A.E. Nelson, Phys. Rev. Lett. 82, 4971 (1999). doi:10.1103/PhysRevLett.82.4971

38. Q.G. Huang, Y.G. Gong, JCAP 0408, 006 (2004). doi:10.1088/ $1475-7516 / 2004 / 08 / 006$

39. X. Zhang, F.Q. Wu, Phys. Rev. D72, 043524 (2005). doi:10.1103/ PhysRevD.72.043524

40. Z. Chang, F.Q. Wu, X. Zhang, Phys. Lett. B633, 14 (2006). doi:10. 1016/j.physletb.2005.10.095

41. Y.Z. Ma, Y. Gong, X. Chen, Eur. Phys. J. C60, 303 (2009). doi:10. 1140/epjc/s10052-009-0876-7

42. M. Li, X.D. Li, S. Wang, X. Zhang, JCAP 0906, 036 (2009). doi:10. 1088/1475-7516/2009/06/036

43. M. Li, X.D. Li, Y.Z. Ma, X. Zhang, Z. Zhang, JCAP 1309, 021 (2013). doi:10.1088/1475-7516/2013/09/021

44. L. Xu, Phys. Rev. D87, 043525 (2013). doi:10.1103/PhysRevD.87. 043525

45. H.C. Kim, J.W. Lee, J. Lee, Europhys. Lett. 102, 29001 (2013). doi:10.1209/0295-5075/102/29001

46. M. Li, R.-X. Miao (2012). arXiv: 1210.0966

47. M. Li, X.D. Li, J. Meng, Z. Zhang, Phys. Rev. D88(2), 023503 (2013). doi:10.1103/PhysRevD.88.023503

48. P.F. Gonzalez-Diaz, C.L. Siguenza, Nucl. Phys. B697, 363 (2004). doi:10.1016/j.nuclphysb.2004.07.020

49. R. Muller, C.O. Lousto, Phys. Rev. D52, 4512 (1995). doi:10.1103/ PhysRevD.52.4512 